\title{
La gestión estratégica universitaria y su influencia en el posicionamiento del licenciado en Administración de la Universidad Nacional Jorge Basadre Grohmann de Tacna- 2018
}

\author{
The strategic university management and its influence in the positioning of \\ the Licensed in Administration of the National University Jorge Basadre \\ Grohmann of Tacna-2018 \\ Felipe Gómez Cáceres \\ Universidad Nacional Jorge Basadre Grohmann \\ Tacna, Perú \\ fgomezc@unjbg.edu.pe \\ https://orcid.org/0000-0002-1232-3267
}

\section{RESUMEN}

[Presentado: 05/10/2020, Aceptado: 02/02/2021]

La presente investigación se elaboró con el objetivo de determinar en qué medida la gestión estratégica universitaria influye en el posicionamiento del licenciado en Administración de la Universidad Nacional Jorge Basadre Grohmann de Tacna en el año 2018. La investigación fue básica, de carácter descriptivo-explicativo. Se trabajó con una muestra de 574 miembros de la comunidad universitaria entre docentes, administrativos y licenciados en Administración de la UNJBG; quienes a través de la aplicación del cuestionario otorgaron datos para medir las variables del presente estudio. El instrumento para medir las variables constó de 26 ítems; fue diseñado por Lozano (2013), pero adaptado para la presente investigación. Asimismo, cuenta con la validación de expertos, análisis de confiabilidad mediante el Alfa de Cronbach y análisis de correlación por ítems. Luego de la recolección y procesamiento de datos, a través del software estadístico SPSS V.22, se describieron los resultados obtenidos por cada ítem y variable correspondiente. Las hipótesis fueron aceptadas con los resultados validados a través de las pruebas paramétricas conducidas por la prueba de Kolomogorov-Smirnov. Además, la Prueba Chi cuadrado y coeficiencia de contingencia permitieron concluir que existe una influencia "alta" significativa de la gestión estratégica universitaria en el posicionamiento del licenciado en Administración de la Universidad Nacional Jorge Basadre Grohmann de Tacna.

Palabras clave: Gestión estratégica, licenciado en Administración, posicionamiento.

\begin{abstract}
The objective of this research was to determine to what extent university strategic management influences the positioning of the graduate in Administration of the Universidad Nacional Jorge Basadre Grohmann de Tacna in 2018. The investigation was basic, descriptive-explanatory in nature. We work with a sample of 574 members of the university community among teachers, administrators and graduates in Administration of the UNJBG; who through the application of the questionnaire provided data to measure the variables of the present study. The instrument to measure the variables consisted of 26 items; it was designed by Lozano (2013), but was adapted for the present research. Likewise, it has expert validation, reliability analysis using Cronbach's alpha and item correlation analysis. After the data collection and processing, through the SPSS V.22 statistical software, the results obtained for each item and corresponding variable were described. The hypotheses were accepted with the results validated through parametric tests conducted by the Kolomogorov-Smirnov test. In addition, the Chi-square test and contingency coefficient allowed concluding that there is a significant "high" influence of university strategic management on the positioning of the graduate in Administration of the Jorge Basadre Grohmann National University of Tacna.
\end{abstract}

Keywords: Strategic management, management degree, positioning.

Este es un artículo de acceso abierto, distribuido bajo los términos de la Licencia Creative Commons Atribución 4.0 Internacional (CC BY 4.0). https://creativecommons.org/licenses/by/4.0/deed.es 


\section{INTRODUCCIÓN}

Los acelerados cambios en el contexto globalizado de la tecnología, los sistemas de información, la economía y política han llevado a las organizaciones a desarrollar procesos de transformación que conducen a ser competitivas, y responder con productos y servicios de calidad para satisfacer al cliente. Ante esta perspectiva, las estructuras organizacionales de las universidades, en el marco de la nueva Ley Universitaria, Ley $\mathrm{N}^{\circ} 30220$, pasan por un proceso de reordenamiento y reestructuración en sus estructuras organizacionales, y tienen como objetivo fundamental promover el mejoramiento continuo de la calidad educativa de las instituciones universitarias como entes fundamentales del desarrollo nacional, de la investigación y de la cultura.

Ante estas tendencias de cambio, es urgente y necesario implantar en las universidades la gestión estratégica universitaria. Esta se puede definir como la nueva forma de promover acciones estratégicas que permitan aumentar la calidad y creatividad en el desempeño de las funciones universitarias con eficacia, eficiencia y efectividad. La aplicación de las mismas permitirá adaptarse a los nuevos enfoques gerenciales y apoyarse en las nuevas tecnologías, y romper la burocracia y la estructura piramidal para lograr acortar los tiempos en la toma de decisiones en función de mayor eficiencia y productividad.

En este marco referencial, la Universidad Nacional Jorge Basadre Grohmann es una organización de educación superior sometida a un proceso de cambio organizacional, pasando por la revisión de su modelo académico, a fin de liderar el rol preponderante de generar y apoyar a la sociedad y al Estado en la solución de problemas socioeconómicas a través de aportes concretos en la formación de los profesionales licenciados en Administración, con ventajas competitivas para impulsar su desarrollo sostenible.

Bajo el enfoque señalado, se presenta la investigación titulada "La gestión estratégica universitaria y su influencia en el posicionamiento del licenciado en Administración de la Universidad Nacional Jorge Basadre Grohmann de Tacna (UNJBG) en el año 2018", la cual se detalla a continuación.

Se tiene como hipótesis que la gestión estratégica universitaria influiría significativamente en el posicionamiento del licenciado en Administración de la Universidad Nacional Jorge Basadre Grohmann de Tacna en el año 2018.

\section{MATERIALES Y MÉTODOS}

La investigación es de tipo básica, denominada también pura o fundamental, ya que contribuye a la ampliación del conocimiento científico, creando nuevas teorías o modificando las ya existentes. En este sentido, se busca mejorar el posicionamiento del licenciado en Administración de la región Tacna, a través de la gestión estratégica universitaria. Por la profundidad de los conocimientos que se pretende alcanzar, el presente estudio es explicativo, porque se busca responder todos los porqués del objetivo, pretende establecer las causas de los eventos que se estudian y se centra en explicar por qué se relacionan dos o más variables. Es decir, conocer y determinar de qué manera influye la gestión estratégica universitaria en el posicionamiento del licenciado en Administración.

En el presente trabajo se utilizó el método hipotético-deductivo, procedimiento o camino que sigue el investigador para hacer de su actividad una práctica científica. El método hipotético-deductivo tiene varios pasos esenciales: observación del fenómeno a estudiar, creación de una hipótesis para explicar dicho fenómeno, deducción de consecuencias y verificación o comprobación de la verdad de los enunciados deducidos comparándolos con la experiencia.

El diseño de la presente investigación es transeccional-correlacional, tiene como objetivo determinar la influencia entre dos o más variables en un momento determinado. En estos diseños, lo que se mide es la influencia entre variables en un tiempo determinado.

Las investigaciones transeccionales se definen como diseños que recolectan datos en un solo momento, en un tiempo único. Su propósito es describir variables y analizar su incidencia.

M: $\quad$ Muestra 1 (Unidad de análisis)

X: $\quad$ Variable 1: Gestión estratégica universitaria

$\mathbf{O}_{1}$ : $\quad$ Observaciones en X: Resultado de medición 
Y: $\quad$ Variable 2: Posicionamiento del licenciado en Administración

$\mathbf{O}_{2}: \quad$ Observaciones en Y: Resultado de medición

i: $\quad$ Influencia

La población de la investigación está conformada por los docentes universitarios, los trabajadores administrativos y los licenciados en Administración de la UNJBG como egresados de la promoción desde el año 2005 al año 2017, siendo un total de 1230. Considerando que la población era un grupo muy extenso, se aplicó el muestro sistemático, obteniendo una muestra de 574 miembros.

Al respecto, Caballero (2000) menciona que un plan de investigación no necesariamente es mejor si considera la técnica más moderna o avanzada o si consideran más técnicas, sino aquella que el investigador considera la más adecuada para sus variables o, más precisamente, para recolectar los datos de los dominios de sus variables. Además, menciona que según la naturaleza de cada variable se determina la técnica, y cada técnica tiene un tipo de instrumento. Para la presente investigación, se utilizó como técnica la encuesta, como la más conveniente para recoger la opinión de la muestra. El instrumento que permitió medir las variables identificadas en la presente investigación fue construido por Lozano (2013) en su investigación relacionada a la gestión estratégica universitaria y posicionamiento del contador público. El instrumento fue ajustado por el investigador. Asimismo, para la confiabilidad o fiabilidad del instrumento se aplicó la validez de expertos y el coeficiente de Alpha de Cronbach.

\section{RESULTADOS}

Después de haber analizado mediante una estadística descriptiva se aplicó un método estadístico que permitió plantear lo siguiente:

$\mathbf{H}_{\mathbf{0}}$ : La gestión estratégica universitaria no influye significativamente en el posicionamiento del licenciado en Administración de la Universidad Nacional Jorge Basadre Grohmann de Tacna en el año 2018.

$\mathbf{H}_{1}$ : La gestión estratégica universitaria influye significativamente en el posicionamiento del licenciado en Administración de la Universidad Nacional Jorge Basadre Grohmann de Tacna en el año 2018.

Nivel de significancia: $\alpha=0.05$

Estadístico de prueba: Chi cuadrado

$$
\begin{aligned}
& X^{2}=\sum \frac{\left(o_{i}-e_{i}\right)^{2}}{e_{i}} \\
& o_{i}=\text { Frecuenciaobservada } \\
& e_{i}=\text { Frecuenciaesperada }
\end{aligned}
$$

\section{Esquema}

- Grados de libertad: $\mathrm{gl}=4$

- Chi cuadrado de la tabla: $X^{2}=9.49$

- Regla de decisión: Rechazar la hipótesis nula $\left(\mathrm{H}_{0}\right)$ si el Chi cuadrado calculado es mayor que el de la tabla.

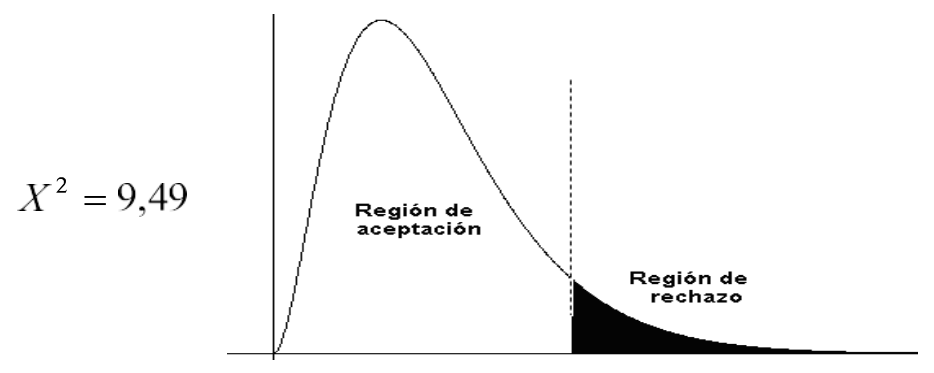




\section{Cálculo del estadístico}

Cálculo efectuado en el programa SPSS

Tabla 1

Pruebas de chi-cuadrado

\begin{tabular}{l|l|l|l}
\hline & Valor & gl & Sig. asintótica (2 caras) \\
\hline Chi-cuadrado de Pearson & $133.460^{\mathrm{a}}$ & 4 & .000 \\
Razón de verosimilitud & 80.567 & 4 & .000 \\
Asociación lineal por lineal & 54.059 & 1 & .000 \\
N de casos válidos & 574 & & \\
\hline Nota: a. 2 casillas (22.2\%) han esperado un recuento menor que 5. El recuento mínimo \\
esperado es 1.20.
\end{tabular}

\section{Coeficiente de contingencia}

El coeficiente de contingencia es un índice estadístico que permite medir la fuerza de la relación existente entre dos variables. Su valor máximo depende del tamaño de la tabla; por esta razón, estos coeficientes solo se pueden comparar entre sí cuando proceden de tablas del mismo tamaño. En el caso de un grado de libertad (tablas 2x2), su valor máximo es 0.707 ; en tablas $3 \times 3$, su valor máximo es 0.816 . Considerando como promedio un valor máximo de 0.8 , se tiene la siguiente escala de grado de influencia.

Tabla 2

Escala de grado de influencia

\begin{tabular}{|c|c|c|c|c|c|}
\hline & $\begin{array}{l}\text { Influencia } \\
\text { mínima }\end{array}$ & $\begin{array}{c}\text { Influencia } \\
\text { baja }\end{array}$ & $\begin{array}{l}\text { Influencia } \\
\text { moderada }\end{array}$ & Influencia alta & $\begin{array}{c}\text { Influencia } \\
\text { muy alta }\end{array}$ \\
\hline 0 & 0.16 & 0.32 & 0.48 & 0.64 & 0.8 \\
\hline
\end{tabular}

Teniendo en consideración la base de datos de ambas variables, se procede a través del SPSS V.22 a determinar el coeficiente de contingencia.

Tabla 3

Medidas simétricas

\begin{tabular}{ll|l|l}
\hline & & Valor & Aprox. Sig. \\
\hline Nominal por Nominal & $\begin{array}{l}\text { Coeficiente } \\
\text { contingencia }\end{array}$ & de & .617 \\
N de casos válidos & & 574 & .000 \\
\hline
\end{tabular}

\section{Justificación y decisión estadística}

Como el estadístico chi cuadrado calculado es mayor al valor crítico obtenido de tabla (133.460 > 9.49), entonces se rechaza la hipótesis nula y se acepta la hipótesis alternativa. Este resultado permite comprobar con un nivel de confianza del $95 \%$ y un Sig o p-valor $<0.05$ que la GESTIÓN ESTRATÉGICA UNIVERSITARIA influye significativamente en el POSICIONAMIENTO del licenciado en Administración de la Universidad Nacional Jorge Basadre Grohmann de Tacna, categorizando la influencia como una influencia alta con un coeficiente de contingencia es 0.617 . 


\section{DISCUSIÓN}

La presente investigación es definida como básica, descriptiva-explicativa, que busca mejorar el posicionamiento del licenciado en Administración de la región Tacna a través de la gestión estratégica universitaria, por lo que se partió con el objetivo de determinar la influencia de la gestión estratégica universitaria en el posicionamiento del licenciado en Administración de la Universidad Nacional Jorge Basadre Grohmann de Tacna en el año 2018. Esto conllevó a tomar en consideración lo señalado por Betancourt (2006), quién señala que la gestión estratégica es “... el arte y/o ciencia de anticipar y gerenciar participativamente en el cambio con el propósito de crear permanentemente estrategias que permitan garantizar el futuro del negocio. A esto agrega como sus elementos relevantes a la planificación, organización, dirección y control. Al respecto, Kotler (2003), uno de los más grandes gurús del marketing, señala que gracias a Ries y Trout, el concepto "posicionamiento" entró a formar parte del vocabulario de marketing en 1982, cuando escribieron Posicionamiento: La batalla por su mente. En realidad, la palabra había sido usada anteriormente en relación con la colocación de los productos en las tiendas, con esperanza de que fuera a la altura de los ojos. Sin embargo, Ries y Trout dieron un giro a este término: "Posicionamiento no es donde posiciona un producto en el lineal. Posicionamiento es la actuación sobre la mente del consumidor", estos argumentos permitieron plantear como hipótesis de la investigación que "la gestión estratégica universitaria influye significativamente en el posicionamiento del licenciado en Administración de la Universidad Nacional Jorge Basadre Grohmann de Tacna en el año 2018". Condición que se comprobó mediante pruebas estadísticas, que efectivamente dieron una categorización de "alta influencia", considerando que el coeficiente de contingencia es 0.617 , encontrándose dentro de un rango de 0 a 0.8 de influencia mínima e influencia máxima respectivamente. Esto permite ratificar lo señalado por Merino (2008) y Fuentes y Vargas (2010) en sus investigaciones, las cuales tienen características similares, por la selección de la muestra y las posibles relaciones con las variables definidas; y que además, bajo otro diseño y nivel de investigación, determinan que la gestión estratégica se aplica en las universidades y es fundamental para tener un óptimo posicionamiento de los egresados y/o estudiantes.

\section{CONCLUSIONES}

Se determinó a un nivel de confianza del $95 \%$ que la gestión estratégica universitaria influye significativamente en el posicionamiento del licenciado en Administración de la Universidad Nacional Jorge Basadre Grohmann de Tacna; categorizado como una influencia alta con un coeficiente de contingencia de 0.617.

Se estableció que la planificación de la gestión estratégica universitaria no es adecuada debido a que no se cumplen con los objetivos y metas de la planificación estratégica de la Universidad. En consecuencia, influye significativamente en el posicionamiento del licenciado en Administración de la Universidad Nacional Jorge Basadre Grohmann de Tacna en el año 2018.

Según el resultado de la investigación, se establece que la organización de la gestión estratégica universitaria no es adecuada, debido a que no se observa una adecuada asignación de tareas, funciones, líneas de autoridad, responsabilidad y coordinación, por tanto, influye en el posicionamiento del licenciado en Administración de la Universidad Nacional Jorge Basadre Grohmann de Tacna en el año 2018.

Se estableció que la dirección de la gestión estratégica universitaria es regular, debido a que se aplica parcialmente el liderazgo, motivación y trabajo en equipo. En consecuencia, influye en el posicionamiento del licenciado en Administración de la Universidad nacional Jorge Basadre Grohmann de Tacna en el año 2018.

Se determinó que el control de la gestión estratégica universitaria es regular, debido a que no está implantado el control interno para evaluar y comparar adecuadamente el desempeño real de las autoridades universitarias frente a los objetivos y metas planeados, por tanto, influye en el posicionamiento del licenciado en Administración de la Universidad Nacional Jorge Basadre Grohmann de Tacna en el año 2018. 


\section{REFERENCIAS}

Caballero, A. (2000). Metodología integral innovadora para planes y tesis. UNAM- Learning editores.

Fuentes, T. y Vargas, D. (2010). Caracterización y posicionamiento del mercado laboral de los egresados del programa de administración de empresas de la universidad de la Salle en Bogotá 2004-2008 [Tesis. Universidad de la Salle].

Kotler, P. (2003). Fundamentos del marketing. Editorial Pearson.

Ley 30220 (2014). Ley Universitaria. Diario Oficial El Peruano. Lima, Perú, 3 de julio del 2014.

Lozano, P. (2013). Gestión Estratégica Universitaria para mejorar el posicionamiento del Contador Público del Centro del Perú [Tesis. Universidad de San Martin de Porres, Lima].

Merino, M. (2008). Diseño de un plan de implementación de la estrategia de posicionamiento de la Universidad Católica Santo Toribio de Mogrovejo en la ciudad de Chiclayo [Tesis. Universidad Mayor de San Marcos, Lima]. 